\title{
Discrete modeling of ion transport and crystallization in layered porous media during drying
}

\section{Rahimi, A. ${ }^{a^{*}}$; Metzger, T. ${ }^{\text {; }}$ Kharaghani, A. ${ }^{\text {a }}$ Tsotsas, E. ${ }^{\text {a }}$}

${ }^{a}$ Chair of Thermal Process Engineering, Otto von Guericke University of Magdeburg, Germany.

${ }^{\mathrm{b}}$ Chemical and Process Engineering, BASF SE, Ludwigshafen, Germany.

*E-mail of the corresponding author: arman.rahimi@ovgu.de

\begin{abstract}
In this work, an isothermal pore network model has been utilized to investigate ion transport and crystallization in layerd porous media during drying. Said network consists of two distinct layers each with a different pore size distribution. One-dimensional approximation at the throat level describes transport phenomena for liquid, vapor, and dissolved salt. An explicit time stepping scheme has been used to obtain fluid pressure fields and ion concentration. Various simulations are carried out which indicate the effect of mean pore size disparity in the top and bottom layer, as well as the effect of drying rate on final crystal distribution.
\end{abstract}

Keywords: pore network modeling, composite material, drying porous media, crystallization, ion transport. 


\section{Introduction}

Layered materials are among the most essential products influencing our lives ranging from everyday use to construction and industrial processes. Painted surfaces, interior wallpapers, composite materials, and fuel cell membrane-electrode combination are among many examples of layered materials. Depending on the application these layered materials may be exposed to a solution (or suspension) at one or several stages of their lifetime. Additionally in the majority of cases said exposure is accompanied by drying that has varying effects which also depends on production and applications of these materials. To this goal, it is paramount to study and identify factors affecting each scenario. In order to provide more context as to which parameters influence precipitation pattern several studies will be looked into in this section.

Preservation of masonry and cultural heritage is an important area where undesired crystallization within porous material is to be avoided. In this framework Pel et al. ${ }^{[1]}$ studied one-sided drying of fire-clayed bricks contaminated by salt solution. In that work, nuclear magnetic resonance technique was used to measure moisture and $\mathrm{NaCl}$ content within the material. In the end it has been concluded that in historical objects generally the formation of efflorescence cannot be avoided. In a similar study the effect of crystallization inhibitors, whose function is to allow a higher degree of supersaturation before nucleation, on drying of fired-clay bricks and Granada limestone is studied by Gupta et al. ${ }^{[2]}$. Satisfactory results were achieved as the mass percentage of the nondestructive efflorescence has been dramatically elevated in the presence of inhibitors. Norouzi Rad et al. ${ }^{[3]}$ studied the pattern of efflorescence formation depending on the spatial distribution pores sized at the surface of a packing of sand grains. Observations indicate that salt crystals are more inclined to form at the small pores as they stay wet for a longer time during drying as pores are sufficiently large to avoid blockage. That study points to the importance of porous media heterogeneity in crystal formation.

With sufficient context on drying induced crystallization in porous media, several studies on crystallization in layered porous structures will be reviewed here. One-sided drying of plaster/substrate systems, where evaporation happens always at the plaster surface, was studied through various experiments by Petković et al. ${ }^{[4]}$. Small pores are at the evaporating side when plaster is paired with Bentheimer sandstone. This leads to the majority of salt crystals to form at the surface or within the plaster layer. On the other hand, in a plaster/calcium-silicate brick system (smaller pores located in the substrate) although some salt crystallized in the plaster layer, a significant amount of crystals was found within the brick. The aforementioned nuclear magnetic resonance technique is used to study the effect of paints on drying behavior of plaster/substrate systems and stone specimens by Diaz Gonçalves et al. ${ }^{[5]}$. It is concluded that crystallization pattern varies depending on many variables such as the pore size of the interior region, hydrophobicity of the paint, and the 
salt itself. This indicates that for such systems any kind of predictive statement should be limited to the specific framework and conclusions should be drawn very cautiously.

In this work, a 3D pore network represents the void space of the porous material. The bilayered structure is modeled by two networks stacked on top of each other leading to a socalled "near surface” (top) and "interior” (bottom) region. Each region has its distinct pore size distribution which is sampled from a normal distribution. The entire network is initially filled with salt solution and evaporation takes place from the top. Simulations are carried out for various combinations of pore size distribution in the bottom and top layer as well as various drying rates. For the sake of comparison the top layer is kept identical among all simulations. Results are presented as solid deposit mass as well as saturation profiles.

\section{Model description}

The selected pore network model consists of two different types of pore geometry. The volume of the liquid in the network is located in the cylindrical pore-throats which are positioned perpendicular to one another. The connection between the throats is established via pore-nodes with no volumes. The nodes provide the computational gird required for vapor and liquid pressure calculation.

\subsection{Pore network drying model}

Drying is considered only from the top, therefore the vapor flow out of the network is modeled in a diffusive boundary layer above the network. The liquid and vapor flow in the entire network are considered quasi-stationary within the network time-steps. It is important to mention that all colligative properties of the salt solution is neglected or in other words the liquid is considered as pure water for liquid flow calculations. This leads to variation of several physical properties, in the course of drying, to be neglected. Said physical parameters include: liquid-gas surface tension, vapor pressure, liquid viscosity, etc.

Vapor diffusion within each throat in the gas-side network is calculated using Stefan correction coupled with the isothermal conditions. The equation can be written as follows:

$$
\dot{\mathrm{M}}_{\mathrm{v}, \mathrm{ij}}=\mathrm{A}_{\mathrm{ij}} \frac{\delta}{\mathrm{L}} \frac{\mathrm{P} \widetilde{\mathrm{M}}_{\mathrm{v}}}{\widetilde{\mathrm{R} T}} \ln \left(\frac{\mathrm{P}-\mathrm{p}_{\mathrm{v}, \mathrm{i}}}{\mathrm{P}-\mathrm{p}_{\mathrm{v}, \mathrm{j}}}\right),
$$

where $\delta$ is the binary diffusion coefficient between water vapor and air $\left(\mathrm{m}^{2} / \mathrm{s}\right), \mathrm{A}_{\mathrm{ij}}$ and $\mathrm{L}_{\mathrm{ij}}$ the throat cross-sectional area and length $\left(\mathrm{m}^{2}\right.$ and $\mathrm{m}$ ) respectively, $\mathrm{P}$ the gas pressure $(\mathrm{Pa}), \mathrm{T}$ the air temperature $(\mathrm{K}), \widetilde{\mathrm{M}}_{\mathrm{v}}$ molar mass of water $(\mathrm{kg} / \mathrm{kmol}), \widetilde{\mathrm{R}}$ universal gas constant $(\mathrm{J} / \mathrm{kmolK})$, and $\mathrm{p}_{\mathrm{v}}$ partial pressure of water in the gas phase. 
The vapor pressure field is calculated by using Equation (1) in mass conservation law for every gas pore with unknown vapor pressure coupled with pores at $\mathrm{P}_{\mathrm{v}}{ }^{*}$ and $\mathrm{P}_{\mathrm{v}, \infty}$ as boundary conditions, which leads to a system of algebraic equation.

Liquid flow rate in the network is governed by the Hagen-Poisueille equation which is written for a throat as follows:

$$
\dot{\mathrm{M}}_{\mathrm{w}, \mathrm{ij}}=\frac{\pi \mathrm{r}_{\mathrm{ij}}^{4}}{8 \mu_{\mathrm{w}} \mathrm{L}}\left(\mathrm{P}_{\mathrm{w}, \mathrm{i}}-\mathrm{P}_{\mathrm{w}, \mathrm{j}}\right),
$$

where $r_{i j}$ represents the throat radius $(\mathrm{m}), \mu_{\mathrm{w}}$ the dynamic viscosity of water $(\mathrm{Pa} \cdot \mathrm{s})$, and $\mathrm{P}_{\mathrm{w}}$ the liquid pressure at the nodes. Similar calculation procedure as for the vapor pressure is considered here for liquid pressure field. The boundary conditions are defined as pressure level for moving menisci and liquid flow rate for stationary menisci. More detailed discussion on the basic drying model can be obtained in Metzger et al. ${ }^{[4]}$.

\subsection{Ion migration model}

Salt concentration in liquid throats in the network is modeled by a $1 \mathrm{D}$ advection-diffusion equation. Advection term is assumed to follow a plug flow and Fick's law governs diffusion in the throats. Discretizing said equation over the space in the throat-node geometry leads to the following:

$$
\frac{d C_{i j}}{d t}=U\left(\frac{C_{i}-C_{i j}}{L_{i j}}\right)+2 D \frac{C_{i}-2 C_{i j}+C_{j}}{L_{i j}{ }^{2}},
$$

where $C$ is the concentration of salt in the solution $\left(\mathrm{kg} / \mathrm{m}^{3}\right)$, $U$ the superficial velocity of liquid in the element $(\mathrm{m} / \mathrm{s})$, and $\mathrm{D}$ the binary diffusion coefficient between solute and solvent $\left(\mathrm{m}^{2} / \mathrm{s}\right)$. Indices $\mathrm{i}$ and $\mathrm{j}$ refer to nodes on either side of the throat ij with liquid flow from node i to node $\mathrm{j}$.

\subsection{Crystallization}

During drying, enrichment of salt takes place due to evaporation of solvent. In this model, salt concentration is tracked over time steps and rises as time goes by. At the end of every time step all liquid throats in the network are scanned and throats that exceed a certain degree of supersaturation $\left(\mathrm{C}^{*+}\right)$ are subject to crystallization. In that case the excess amount of salt is precipitated until salt concentration falls below the saturation concentration $\left(\mathrm{C}^{*}\right)$. Therefore, said amount of precipitated salt is calculated as follows:

$$
M_{s, i j}=V_{i j}\left(C_{i}-C^{*+}\right)
$$

It is assumed that the time scale for crystallization is significantly shorter than that of the ion migration which leads to an instant crystallization model in this case. 


\section{Simulation results}

Pore network simulations are carried out with a 3D cubic pore network comprised of 15 (lateral) $\times 15$ (lateral) $\times 19$ (vertical) nodes. This network is constructed as two distinct layers stacked on top of each other with each layer containing 9 counts of slices in the vertical direction. Throats throughout the network have a uniform length of $6 \mu \mathrm{m}$ whereas their radii are sampled from a different normal distribution function for each layer. The top layer is kept identical across all simulations with a mean throat radius of $1000 \mathrm{~nm}$ whereas the bottom layer has 2 different variations presented in Table 1.

Table 1. Throat radii distribution of the bottom layer

\begin{tabular}{lll}
\hline \multicolumn{1}{c}{ network } & \multicolumn{1}{c}{$\mathbf{1}$} & \multicolumn{1}{c}{$\mathbf{2}$} \\
\hline mean radius $(\mathrm{nm})$ & 100 & 1500 \\
standard deviation $(\mathrm{nm})$ & 20 & 150 \\
\hline
\end{tabular}

The physical parameters used in the simulations are shown in Table 2.

Table 2. Physical parameters.

\begin{tabular}{cccccc}
\hline $\boldsymbol{\mu}_{\mathbf{w}}(\mathbf{P a} . \mathbf{s})$ & $\boldsymbol{\rho}_{\mathbf{l}}\left(\mathbf{k g} / \mathbf{m}^{3}\right)$ & $\boldsymbol{\sigma}_{\mathbf{l}}(\mathbf{N} / \mathbf{m})$ & $\boldsymbol{\delta}\left(\mathbf{m}^{2} / \mathbf{s}\right)$ & $\mathbf{D}\left(\mathbf{m}^{2} / \mathbf{s}\right)$ & $\mathbf{C}^{*}\left(\mathbf{k g} / \mathbf{m}^{3}\right)$ \\
\hline $1 \times 10^{-3}$ & $1.27 \times 10^{3}$ & $7.2 \times 10^{-2}$ & $2.5 \times 10^{-5}$ & $1 \times 10^{-9}$ & $1 \times 10^{2}$ \\
\hline
\end{tabular}

\subsection{Network 1}

Network 1 is comprised of two layers with monomodal pore size distributions. In this case the bottom layer has smaller throats compared to the top layer. Final crystal mass profile obtained by slice averaging is presented in Fig. 1 for 3 different drying rates. These various drying rates are simulated by adjusting the value of saturation vapor pressure $\left(\mathrm{P}_{\mathrm{v}}{ }^{*}\right)$ until the desired drying times, as shown in Table 3, are achieved.

Table 3. Drying rate date

\begin{tabular}{llll}
\hline \multicolumn{1}{c}{ drying rate } & slow & moderate & fast \\
\hline $\mathrm{P}_{\mathrm{v}}{ }^{*}(\mathrm{~Pa})$ & 160 & 1600 & 7384 \\
drying time $(\mathrm{s})$ & 1200 & 120 & 24 \\
\hline
\end{tabular}

Low initial salt concentration of $10 \mathrm{~kg} / \mathrm{m}^{3}$ is considered for the first set of simulations which provides enough room for ion diffusion in the liquid before saturation concentration is reached. It is observed that the profile for the case of slow drying (Fig. 1 (a)) is far more homogeneous and the gradient increases as drying rate speeds up. Moreover, looking directly at the first slice (at the surface) it can be seen that almost double the amount of crystals are found at the surface for the case of fast drying (Fig. 1 (c)) compared to slow drying. Another important factor that can play an important role depending on the 
application is the amount of solid deposit found at the interface between the two layers. This value seems to remain unchanged between these sets of simulation yet it is quantified as a moderately low amount.

Fig. 1. Final crystal mass profile of network 1 with initial salt concentration of $10 \mathrm{~kg} / \mathrm{m}^{3}$ for (a)

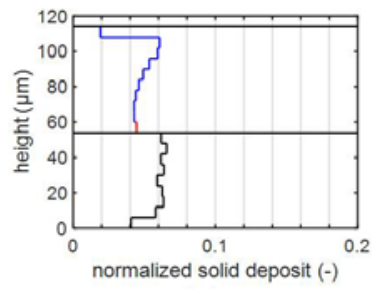

(a)

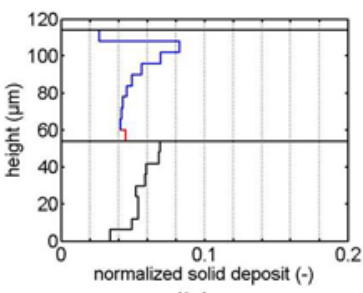

(b)

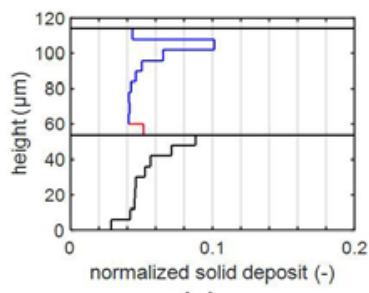

(c)

slow drying, (b) moderate drying, and (c) fast drying.

Elevating the initial salt concentration to $90 \mathrm{~kg} / \mathrm{m}^{3}$ (very close to saturation) drastically alters the final solid distribution in the system. It can be seen that such high initial concentration eliminates final gradient in crystal distribution except for the top and interfacial slices (Fig. 2).

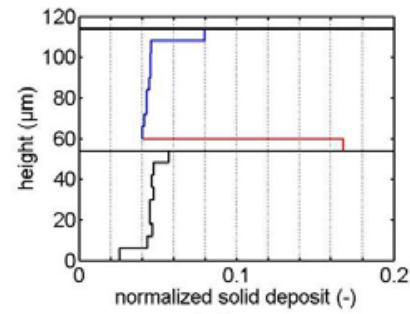

(a)

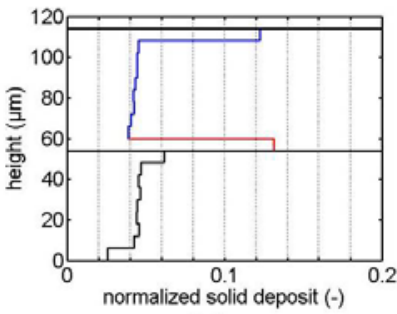

(b)

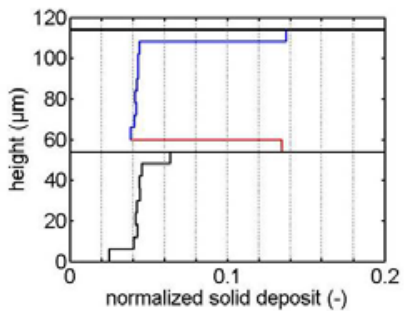

(c)

Fig. 2. Final crystal mass profile of network 1 with initial salt concentration of $90 \mathrm{~kg} / \mathrm{m}^{3}$ for (a) slow drying, (b) moderate drying, and (c) fast drying.

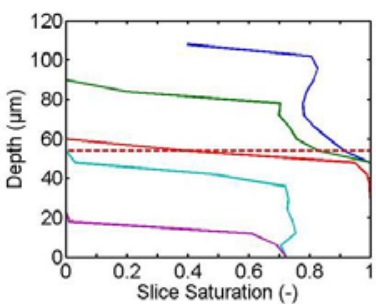

(a)

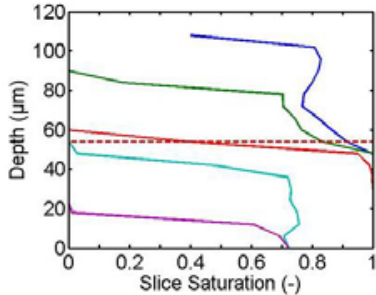

(b)

Fig. 3. Saturation proflies in the course of drying for network one obtained from simulations with (a) slow drying and (b) fast drying. 
Additionally the profiles look much more alike for the three different drying rates. These effects are attributed to the lack of ion diffusion caused by high initial concentration. This significant similarity also hints at lack of change in the liquid transport regime between for the various drying rates. This has been confirmed by comparing saturation proflies for the cases of slow and fast drying which look close to identical (Fig. 3).

\subsection{Network 2}

The final set of simulations are carried out with network 2 where the bottom layer has considerable larger throats and the top layer. In this case the trend of crystal mass profile for varying drying rate is very similar to that of the network 1 as no viscous stabilization of the drying front is achieved. Therefore a comparison of network 1 and 2 is presented here which is more fruitful.

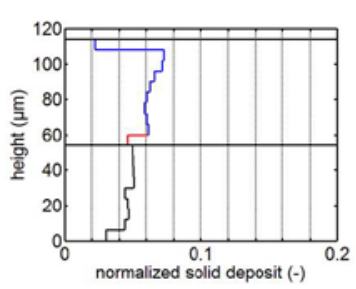

(a)

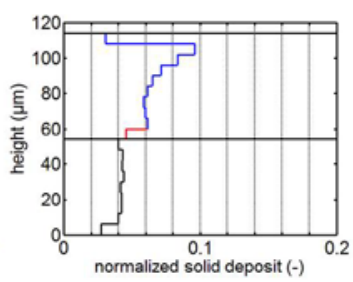

(b)

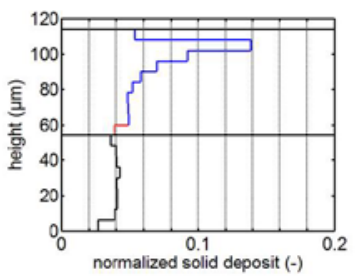

(c)

Fig. 4. Final crystal mass profile of network 2 with initial salt concentration of $10 \mathrm{~kg} / \mathrm{m}^{3}$ for (a) slow drying, (b) moderate drying, and (c) fast drying.

The important characteristic of the network 2 is that once breakthrough to the bottom layer takes place in the absence of viscous stabilization the majority of the evaporated liquid is supplied by large throats in the bottom layer due to high capillary pressure. This continues until the connection between the liquid in the top and bottom layer is lost and capillary pumping is not possible. This behavior is completely opposite to that of the network 1 (Fig. 1) where almost no capillary pumping from the bottom layer to the top layer is possible. This explains the significantly lower amount of solid deposit in the bottom layer and the elevated amount in the top layer for network 2 (Fig. 4). The fact that liquid connection is almost entirely lost at the early stages of drying explains lack of crystal mass gradient in the bottom layer as ion back diffusion from evaporation site to the this region is not possible.

The results for increased concentration demonstrate significant accumulation of crystals at the top slice (Fig. 5) which was also observed for network 1 (Fig. 2). However it is the clear difference between the two networks is observed at the interface of the two layers. Almost no accumulation of crystals at the interface is observed for network 2 (Fig 5). The accumulation that is observed at the interface for network 1 is due to the fact that the bottom is almost fully saturated as the top layer dries out (a result of small pores with low capillary pressure). This means that as the drying front arrives at the interface, the bottom 
layer starts to dry in a similar fashion as to the top layer. In case of network 2 at the moment that drying front reaches the interface the liquid connection in the bottom layer has already been lost (due to capillary pumping to the surface). Consequently recession of drying front continues without a halt to the end of drying.

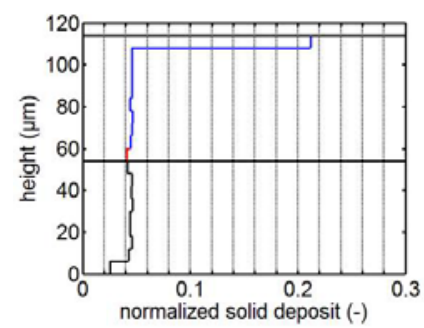

(a)

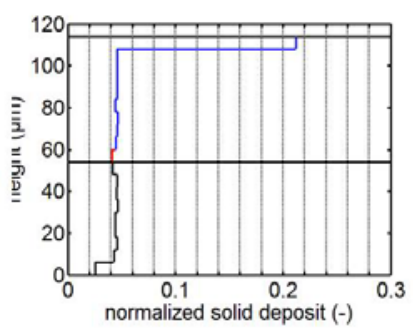

(b)

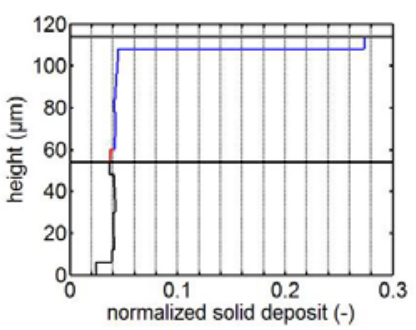

(c)

Fig. 5. Final crystal mass profile of network 2 with initial salt concentration of $90 \mathrm{~kg} / \mathrm{m}^{3}$ for (a) slow drying, (b) moderate drying, and (c) fast drying.

\section{Conclusions}

In this work, a 3D pore network model has been utilized to run simulations for drying of layered porous materials in the presence of dissolved components. The majority of salt crystals are accumulated in the top layer for network with large pores in the bottom. For low concentration similar amount of crystals is obtained in both layers for network with smaller pores in the bottom. Moreover, enrichment at the interface between two layers is achieved only with high initial concentration and network with smaller pores in the bottom layer.

\section{References}

[1] Pel, L; Huinink, H.; Kopinga, K. Ion transport and crystallization in inorganic building materials as studied by nuclear magnetic resonance. Applied Physics Letters 2002, 81(15), DOI 10.1063/1.1512326.

[2] Petković, J.; Huinink, H.; Pel, L.; Kopinga, K.; van Hees, R. P. J. Salt transport in plaster/substrate layers. Materials and Structures 2007, 40, 475-495.

[3] Gupta, S.; Terheiden, K.; Pel, L.; Sawdy, A. Influence of ferrocyanide inhibitors on the transport and crystallization processes of sodium chloride in porous building materials. Crystal Growth \& Design 2012, 12(8), 3888-3898.

[4] Gonçalves, T. D.; Pel, L.; Rodrigues, J. D. Influence of paitns on drying and salt distribution processes in porous building materials. Construction and Building Materials 2009, 23(5), 1751-1759, Compatibality of Plasters and Renders on Salt Loaded Substrates.

[5] Metzger, T.; Irawan, A.; Tsotsas, E. Isothermal Drying of Pore Networks: Influence of Friction for Different Pore Structures. Drying Technology 2007, 25, 49-57.

[6] Norouzi Rad, M.; Shokri, N.; Sahimi, M. Pore-scale dynamics of salt precipitation in drying porous media. Physical Review E, 2013, 88:032404. 